\title{
QUASI-INTERIOR IDEALS AND WEAK-INTERIOR IDEALS
}

\author{
M. MURALI KRISHNA RAO \\ Department of Mathematics, GIT, GITAM University, Visakhapatnam- 530 045, Andhra Pradesh, India \\ Corresponding author: mmarapureddy@gmail.com
}

Received Dec. 10, 2019

\begin{abstract}
АвsтRACт. In this paper, as a further generalization of ideals, we introduce the notion of quasiinterior ideals and weak -interior ideals as a generalization of quasi ideal, bi-ideal and interior ideal of semigroups.We study the properties of weak-interior ideals of semigroup and characterize the weak-interior simple semigroup, regular semigroup using quasi-interior ideals weak-interior ideal ideals of semigroups.

2010 Mathematics Subject Classification. 20M17, 06F05.

Key words and phrases. quasi ideal; bi-ideal; interior ideal; quasi-interior ideal; weak-interior ideal; bi-quasi ideal; regular semigroup; quasi-interior simple semigroup.
\end{abstract}

\section{InTRODUCTION}

Semigroup, as the basic algebraic structure was used in the areas of theoretical computer science as well as in the solutions of graph theory, optimization theory and in particular for studying automata, coding theory and formal languages.

The notion of ideals was introduced by Dedekind for the theory of algebraic numbers, was generalized by Noether for associative rings. The one and two sided ideals introduced by her, are still central concepts in ring theory and the notion of a one sided ideal of any algebraic structure is a generalization of notion of an ideal. The notion of the bi-ideal in semigroups is a special case of $(\mathrm{m}, \mathrm{n})$ ideals. In 1952, the concept of bi-ideals for semigroup was introduced by Good and Hughes [1] and the notion of bi-ideals in associative rings was introduced by Lazos and Szasz $[8,9,10]$. We know that the notion of a one sided ideal of any algebraic structure is a generalization of notion of an ideal. The quasi ideals are generalization of left ideals and right

DOI: $10.28924 /$ APJM/7-21 
ideals whereas the bi-ideals are generalization of quasi ideals. Steinfeld [30] first introduced the notion of quasi ideals for semigroups and then for rings. Iseki [3.4.5] introduced the concept of quasi ideal for a semigroup. Quasi ideals in $\Gamma$ - semigroups were studied by Jagtap and Pawar [7]. Murali Krishna Rao et al. [11-29] studied ideals,derivations and properties of $\Gamma$ - incline and $\Gamma$-semiring.

In this paper, as a further generalization of ideals, we introduce the notion of quasi-interior ideal, weak-interior ideal and study the properties of quasi-interior ideals and weak-interior ideal of semigroup, characterization of simple semigroup and regular semigroup using quasiinterior ideals and weak-interior ideal of semigroup.

\section{Preliminaries}

In this section, we will recall some of the fundamental concepts and definitions, which are necessary for this paper.

Definition 2.1. A semigroup is an algebraic system $(S,$.$) consisting of a non-empty set S$ together with an associative binary operation ". .

Definition 2.2. A subsemigroup $T$ of $S$ is a non-empty subset $T$ of $S$ such that $T T \subseteq T$.

Definition 2.3. A non-empty subset $T$ of $S$ is called a left (right) ideal of $S$ if $S T \subseteq T(T S \subseteq T)$.

Definition 2.4. A non-empty subset $T$ of $S$ is called an ideal of $S$ if it is both a left ideal and a right ideal of $S$.

Definition 2.5. A non-empty subset $Q$ of $S$ is called a quasi ideal of $S$ if $Q S \cap S Q \subseteq Q$.

Definition 2.6. A subsemigroup $T$ of $S$ is called a bi-ideal of $S$ if $T S T \subseteq T$.

Definition 2.7. A subsemigroup $T$ of $S$ is called an interior ideal of $S$ if $S T S \subseteq T$.

Definition 2.8. An element $a$ of a semigroup $S$ is called a regular element if there exists an element $b$ of $S$ such that $a=a b a$.

Definition 2.9. A semigroup $S$ is called a regular semigroup if every element of $S$ is a regular element.

Definition 2.10. Let $S$ be a semigroup. An element $1 \in S$ is said to be unity if $x 1=1 x=x$ for all $x \in S$. 
Definition 2.11. A subsemigroup $A$ of $S$ is called a left( right) bi- quasi ideal of $M$ if $S A \cap$ $A S A(A S \cap A S A) \subseteq A$.

Definition 2.12. A subsemigroup $A$ of $S$ is called a bi- quasi ideal of $M$ if $A$ is a left bi- quasi ideal and a right bi- quasi ideal of $M$

Definition 2.13. A semigroup $M$ is a left (right) simple semigroup if $M$ has no proper left (right) ideal of $M$

Definition 2.14. A semigroup $M$ is a bi-quasi simple semigroup if $M$ has no proper bi-quasi ideal of $M$

Definition 2.15. A semigroup $M$ is said to be simple semigroup if $M$ has no proper ideals.

\section{QUASI-INTERIOR IDEALS OF SEMIGROUPS}

In this section, we introduce the notion of left quasi-interior ideal as a generalization of bi-ideal,quasi-ideal and interior ideal of semigroup and study the properties of quasi- interior ideal of semigroups.

Definition 3.1. A non-empty subset $B$ of a semigroup $M$ is said to be left quasi- interior ideal of $M$ if $B$ is a subsemigroup of $M$ and $M B M B \subseteq B$

Definition 3.2. A non-empty subset $B$ of $M$ is said to be right quasi- interior ideal of $M$ if $B$ is a subsemigroup of $M$ and $B M B M \subseteq B$

Definition 3.3. [23] Let $M$ be a semigroup. $L$ is said to be quasi- interior ideal of $M$ if it is both a left quasi- interior ideal and a right quasi- interiori ideal of $M$.

Everyleft quasi- interior ideal of a semigroup $M$ need not be bi-ideal,quasi-ideal, interior ideal quasi-interior ideal. and bi-quasi ideals of semigroup $M$.

Example 3.4. Let $N$ be a set of all natural numbers. $N$ is additive abelian semigroup with respect to usual addition of integers. A subset $I=2 N$ of $N$ is a left quasi- interior ideal of $N$ but not bi-ideal,quasi-ideal, interior ideal, quasi-interior ideal and bi-quasi ideal of semigroup $N$.

Theorem 3.5. Every left quasi- interior ideal of a semigroup $M$ is a bi-ideal of semigroup $M$. 
Proof. Let $B$ be a left quasi- interior ideal of the semigroup $M$. Then $M B M B \subseteq B$.

Therefore $B M B \subseteq M B M B \subseteq B$.

Hence every left quasi- interior ideal of the semigroup $M$ is a bi-ideal of semigroup $M$.

Theorem 3.6. Every interior ideal of a semigroup $M$ is a left quasi-interior ideal of $M$.

Proof. Let $I$ be an interior ideal of the semigroup $M$.

Then $M I M I \subseteq M I M \subseteq I$.

Hence $I$ is a left quasi- interior ideal of the semigroup $M$.

In the following theorems, we mention some important properties and we omit the proofs since proofs are straight forward.

Theorem 3.7. Let $M$ be a semigroup. Every left ideal is a left quasi-interior ideal of $M$.

Theorem 3.8. Let $M$ be a semigroup. Every right ideal is a right quasi-interior ideal of $M$.

Theorem 3.9. Let $M$ be a semigroup.Every quasi ideal is a quasi-interior ideal of $M$.

Theorem 3.10. Let $M$ be a semigroup. Every ideal is a left quasi-interior ideal of $M$.

Theorem 3.11. Let $M$ be a semigroup. The intersection of a right ideal and a left ideal of $M$ is a left quasi- interior ideal of $M$.

Theorem 3.12. Let $M$ be a semigroup. If $L$ is a left ideal and $R$ is a right ideal of a semigroup $M$ then $B=R L$ is a quasi-interior ideal of $M$.

Theorem 3.13. Let $M$ be a semigroup. If $B$ is a left quasi-interior ideal and $T$ isa subsemigroupof $M$ then $B \cap T$ is a quasi- interior ideals of semigroup $M$.

Theorem 3.14. Let $M$ be a semigroup and $B$ be a subsemigroup of $M$.If $M M M B \subseteq B$ then $B$ is a left quasi-interior ideal of $M$.

Theorem 3.15. Let $M$ be a semigroup and $B$ be a subsemigroup of $M$.If $M M M B \cap B M M M \subseteq B$ then $B$ is a left quasi-interior ideal of $M$.

Theorem 3.16. Let $M$ be a semigroup and $B$ be a subsemigroup of $M . B$ is a left quasi-interior ideal of $M$ if and only if there exist a left ideal $L$ and a right ideal $R$ of $M$ such that $L R \subseteq B \subseteq R \cap L$. 
Proof. Suppose $B$ is a quasi- interior ideal of the semigroup $M$.

Then $M B M B \subseteq B$. Let $R=B M$ and $L=M B$.

Then $L$ and $R$ are left ideal and right ideal of $M$ respectively.

Therefore $L R \subseteq B \subseteq R \cap L$.

Conversely suppose that there exist $L$ and $R$ aLre left and right ideal of $M$ respectively such that $L R \subseteq B \subseteq R \cap L$.Then

$M B M B \subseteq M(R \cap L) M(R \cap L)$

$\subseteq R M R M L$

$\subseteq R L \subseteq B$

Hence $B$ is a left quasi- interior ideal of the semigroup $M$.

Corollary 3.17. Let $M$ be a semigroup and $B$ be a subsemigroup of $M . B$ is a right quasi-interior ideal of $M$ if and only if there exist a left ideal $L$ and a right ideal $R$ of $M$ such that $R L \subseteq B \subseteq R \cap L$.

Corollary 3.18. Let $M$ be a semigroup and $B$ be a subsemigroup of $M . B$ is a quasi-interior ideal of $M$ if and only if there exist a left ideal $L$ and a right ideal $R$ of $M$ such that $R L \cap L R \subseteq B \subseteq R \cap L$.

Theorem 3.19. The intersection of a left quasi-interior ideal $B$ of a semigroup $M$ and a right ideal $A$ of $M$ is always a left quasi-interior ideal of $M$.

Proof. Suppose $C=B \cap A$.

$$
\begin{aligned}
M C M C & \subseteq M B M B \subseteq B \\
M C M C & \subseteq M A M A \subseteq A, \text { since } A \text { is a left ideal of } M \\
\text { Therefore } M C M C & \subseteq B \cap A=C .
\end{aligned}
$$

Hence the intersection of a left quasi- interior ideal $B$ of the semigroup $M$ and a subsemigroup $A$ of $M$ is always a left quasi- interior ideal of $M$.

Corollary 3.20. The intersection of a quasi-interior ideal $B$ of a semigroup $M$ and an ideal $A$ of $M$ is always a left quasi-interior ideal of $M$.

Theorem 3.21. Let $A$ and $C$ be left quasi-interior ideals of a semigroup $M$ and $B=A C$. If $C C=C$ then $B$ is a left quasi-interior ideal of $M$. 
Proof. Let $A$ and $C$ be left quasi- interior ideals of the semigroup $M$ and $B=A C$. $B B=$ $A C A C=A C C A C \subseteq A M C M C \subseteq A C=B$. Obviously $B=A C$ is a subsemigroup of $M$

$$
\begin{aligned}
M B M B & =M A C M A C \\
& \subseteq M A M A C \subseteq A C=B .
\end{aligned}
$$

Hence $B$ is a left quasi- interior ideal of $M$.

Corollary 3.22. Let $A$ and $C$ be quasi- interior ideals of a semigroup $M$ and $B=C A$. If $C C=C$ then $B$ is a quasi-interior ideal of $M$.

Theorem 3.23. Let $A$ and $C$ be subsemigroups of a semigroup $M$ and $B=A C$. If $A$ is the left ideal then $B$ is a left quasi-interior ideal of $M$.

Proof. Let $A$ and $C$ be subsemigroups of the semigroup $M$ and $B=A C$ Suppose $A$ is the left ideal of $M . B B=A C A C \subseteq A C=B$.

$$
\begin{aligned}
M B M B & =M A C M A C \\
& \subseteq A C=B .
\end{aligned}
$$

Hence $B$ is a left quasi- interior ideal of $M$.

Corollary 3.24. Let $A$ and $C$ be subsemigroups of a semigroup $M$ and $B=A C$. If $C$ is a right ideal then $B$ is a left quasi-interior ideal of $M$.

Theorem 3.25. Let $M$ be a semigroup and $T$ be a non-empty subset of $M$. Then every subsemigroup of $T$ containing $M T M T$ is a left quasi- interior ideal of semigroup $M$.

Proof. Let $B$ be a subsemigroup of $T$ containing $M T M T$. Then

$$
\begin{aligned}
M B M B & \subseteq M T M T \\
& \subseteq B .
\end{aligned}
$$

Therefore $M B M B \subseteq B$.

Hence $B$ is a left quasi- interior ideal of $M$.

Theorem 3.26. $B$ is a left quasi-interior ideal of a semigroup $M$ if and only if $B$ is a left ideal of some ideal $R$ of semigroup $M$. 
Proof. Suppose $B$ is a left ideal of some ideal $R$ of the semigroup $M$.Then $R B \subseteq B, R M \subseteq$ $R$.Hence $M B M B \subseteq M R M B \subseteq R M B \subseteq R B \subseteq B$. Therefore $B$ is a left quasi- interior ideal of a semigroup $M$. Conversely suppose that $B$ is a left quasi- interior ideal of a semigroup $M$ .Then $M B M B \subseteq B$. Therefore $B$ is a left ideal of ideal $M B M$ of the semigroup $M$.

Corollary 3.27. $B$ is a right quasi-interior ideal of a semigroup $M$ if and only if $B$ is a right ideal of some ideal of semigroup $M$.

Theorem 3.28. If $B$ is a left quasi-interior ideal of a semigroup $M, T$ is a sub semigroup of $M$ and $T \subseteq B$ then $B T$ is a left quasi- interior ideal of $M$.

Proof. $B T B T \subseteq B T$.

Hence $B T$ is a subsemigroup of $M$.

$$
\begin{aligned}
& \text { We have } M B T M \subseteq M B M \\
& \qquad \text { and } T M B T \subseteq B M B \\
& \quad \Rightarrow M B T M B T \subseteq M B M B T \subseteq B T .
\end{aligned}
$$

Hence $B T$ is a left quasi- interior ideal of the semigroup $M$.

Theorem 3.29. Let $B$ be bi-ideal of a semigroup $M$ and $I$ be interior ideal of $M$. Then $B \cap I$ is a left quasi-interior ideal of $M$.

Proof. Obviously $B \cap I$ is subsemigroup of $M$. Suppose $B$ is a bi-ideal of $M$ and $I$ is an interior ideal of $M$. Then

$$
\begin{array}{r}
M(B \cap I) M(B \cap I) \subseteq M B M B \subseteq B, \\
M(B \cap I) M(B \cap I) \subseteq M I M I \subseteq I
\end{array}
$$

Therefore $M(B \cap I) M(B \cap I) M \subseteq B \cap I$.

Hence $B \cap I$ is a left quasi- interior ideal of $M$.

Theorem 3.30. Let $M$ be a semigroup and $T$ be a subsemigroup of $M$. Then every subsemigroup of $T$ containing $M T M T$ is a left quasi-interior ideal of $M$.

Proof. Let $C$ be a subsemigroup of $T$ containing $M T M T$. Then

$$
\begin{aligned}
M C M C & \subseteq M T M T \\
& \subseteq C .
\end{aligned}
$$


Hence $C$ is a left quasi- interior ideal of the semigroup $M$.

Theorem 3.31. Let $M$ be a semigroup. If $M=M a$, for all $a \in M$. Then every left quasi-interior ideal of $M$ is a quasi ideal of $M$.

Proof. Let $B$ be a left quasi- interior ideal of the semigroup $M$ and $a \in B$. Then

$$
\begin{aligned}
& M B M B \subseteq B \\
\Rightarrow & M a \subseteq M B \\
\Rightarrow & M \subseteq M B \subseteq M \\
\Rightarrow & M B=M \\
\Rightarrow & B M=B M B \subseteq M B M B \subseteq B \\
\Rightarrow & M B \cap B M \subseteq M M \cap B M \subseteq B .
\end{aligned}
$$

Therefore $B$ is a quasi ideal of $M$. Hence the theorem.

Theorem 3.32. The intersection of $\left\{B_{\lambda} \mid \lambda \in A\right\}$ left quasi-interior ideals of a semigroup $M$ is a quasi- interior ideal of $M$.

Proof. Let $B=\bigcap_{\lambda \in A} B_{\lambda}$. Then $B$ is a subsemigroup of $M$.

Since $B_{\lambda}$ is a left quasi- interior ideal of $M$, we have

$$
\begin{aligned}
& M B_{\lambda} M B_{\lambda} \subseteq B_{\lambda}, \text { for all } \lambda \in A \\
\Rightarrow & M \cap B_{\lambda} M \cap B_{\lambda} \subseteq \cap B_{\lambda} \\
\Rightarrow & M B M B \subseteq B .
\end{aligned}
$$

Hence $B$ is a left quasi- interior ideal of $M$.

Theorem 3.33. Let $B$ be a left quasi-interior ideal of a semigroup $M, e \in B, e B \subseteq B$ and $e$ be an idempotent. Then eB is a left quasi-interior ideal of $M$. 
Proof. Let $B$ be a left quasi- interior ideal of the semigroup $M$. Suppose $x \in B \cap e M$. Then $x \in B$ and $x=e y, y \in M$.

$$
\begin{aligned}
x & =e y \\
& =e e y \\
& =e(e y) \\
& =e x \in e B .
\end{aligned}
$$

Therefore $B \cap e M \subseteq e B$

$$
\begin{aligned}
& e B \subseteq B \text { and } e B \subseteq e M \\
\Rightarrow & e B \subseteq B \cap e M \\
\Rightarrow & e B=B \cap e M
\end{aligned}
$$

Hence $e B$ is a left quasi- interior ideal of $M$.

Corollary 3.34. Let $M$ be a semigroup $M$ and e be an idempotent. Then eM and $M e$ are quasiinterior ideals of $M$.

Theorem 3.35. If $B$ be a left quasi-interior ideal of a semigroup $M$, then $B$ is a left bi-quasi ideal of $M$.

Proof. Suppose $B$ is a bi-quasi interior ideal of the semigroup $M$. Then $B M B \subseteq M B M B$.

Therefore $M B \cap B M B \subseteq B M B \subseteq M B M B \subseteq B$

Hence $B$ is a left bi- quasir ideal of $M$.

Corollary 3.36. If $B$ be a right quasi-interior ideal of a semigroup $M$, then $B$ is a right bi-quasi ideal of $M$.

Corollary 3.37. If $B$ be a quasi-i-interior ideal of a semigroup $M$, then $B$ is a bi-quasi ideal of $M$.

Theorem 3.38. If $B$ be a left quasi-interior ideal of a semigroup $M$, then $B$ is a bi-interior ideal of a semigroup $M$.

Proof. Suppose $B$ is a left quasi- interior ideal of the semigroup $M$. Then $B M B \subseteq M B M B$ Therefore $M B M \cap B M B \subseteq B M B \subseteq M B M B \subseteq B$

Hence $B$ is a bi- interior ideal of $M$. 
Theorem 3.39. Let $B$ be subsemigroup of a semigroup $M$. If $B$ can be represented as $B=L L$, where $L$ is a left ideal of $M$ then $B$ is a left quasi-interior ideal of $M$.

Proof. Suppose $B=L L$, where $L$ is a left ideal of $M$.

$$
\begin{aligned}
M B M B & =M L L M L L \\
& \subseteq L L=B .
\end{aligned}
$$

Hence $B$ is a left Lquasi- interior ideal of the semigroup $M$.

Definition 3.40. A semigroup $M$ is said to be quasi- interior simple semigroup if $M$ has no quasi- interior ideals other than $M$ itself.

Theorem 3.41. Let $M$ be a simple semigroup. Every left quasi-interior ideal is a left ideal of $M$.

Proof. Let $M$ be a simple semigroup and $B$ be a left quasi- interior ideal of $M$.

Then $M B M B \subseteq B$ and $M B M$ is an ideal of $M$.

Since $M$ is a simple semigroup, we have $M B M=M$. Hence

$$
\begin{aligned}
& M B M B \subseteq B \\
\Rightarrow & M B \subseteq B .
\end{aligned}
$$

Hence the theorem.

Theorem 3.42. Let $M$ be a semigroup. $M$ is a quasi-interior simple semigroup if and only if $<a>=$ $M$, for all $a \in M$ and where $<a>$ is the smallest left quasi-interior ideal generated by $a$.

Proof. Let $M$ be a semigroup. Suppose $M$ is a quasi- interior simple semigroup, $a \in M$ and $B=M a$.

Then $B$ is a left ideal of $M$.

Therefore, by Theorem[3.5 ], $B$ is a left quasi- interior ideal of $M$.

Therefore $B=M$. Hence $M a=M$, for all $a \in M$.

$$
\begin{aligned}
& M a \subseteq<a>\subseteq M \\
\Rightarrow & M \subseteq<a>\subseteq M .
\end{aligned}
$$

Therefore $M=<a>$. 
Suppose $\langle a\rangle$ is the smallest left quasi- interior ideal of $M$ generated by $a$ and $<a\rangle=M$ and $A$ is the left quasi- interior ideal and $a \in A$. Then

$$
\begin{gathered}
<a>\subseteq A \subseteq M \\
\Rightarrow M \subseteq A \subseteq M .
\end{gathered}
$$

Therefore $A=M$. Hence $M$ is a quasi- interior simple semigroup.

Theorem 3.43. Let $M$ be a semigroup. Then $M$ is a left quasi-interior simple semigroup if and only if $M a M a=M$, for all $a \in M$.

Proof. Suppose $M$ is left quasi- interior simple semigroup and $a \in M$.

Therefore $M a M a$ is a left quasi- interior ideal of $M$.

Hence $M a M a=M$, for all $a \in M$.

Conversely suppose that $M a M a=M$, for all $a \in M$.

Let $B$ be a left quasi- interior ideal of the semigroup $M$ and $a \in B$.

$$
\begin{aligned}
M & =M a M a \\
& \subseteq B M B M B \subseteq B
\end{aligned}
$$

Therefore $M=B$.

Hence $M$ is a quasi- interior simple semigroup.

Definition 3.44. A semigroup $M$ is a left (right) simple semigroup if $M$ has no proper left (right) ideal of $M$

Definition 3.45. A semigroup $M$ is said to be simple semigroup if $M$ has no proper ideals.

Theorem 3.46. If semigroup $M$ is a left simple semigroup then every left quasi-interior ideal of $M$ is a right ideal of $M$. 
Proof. Let $B$ be a left quasi- interior of the left simple semigroup $M$. Then $M B$ is a left ideal of $M$ and $M B \subseteq M$. Therefore $M B=M$. Then

$$
\begin{aligned}
& M B M=M M \subseteq M \\
\Rightarrow & B M=B M B \\
\Rightarrow & B M=B M B \subseteq M B M B \subseteq B \\
\Rightarrow & B M \subseteq B .
\end{aligned}
$$

Hence every left quasi- interior ideal is a right ideal of $M$.

Corollary 3.47. If semigroup $M$ is a right simple semigroup then every left quasi-interior ideal of $M$ is a left ideal of $M$.

Corollary 3.48. Every left quasi-interior ideal of left and right simple semigroup $M$ is an ideal of $M$.

Theorem 3.49. Let $M$ be a semigroup and $B$ be a left quasi-interior ideal of $M$. Then $B$ is a minimal left quasi-interior ideal of $M$ if and only if $B$ is a left quasi-interior simple subsemigroup of $M$.

Proof. Let $B$ be a minimal left quasi- interior ideal of the semigroup $M$ and $C$ be a left quasiinterior ideal of $B$. Then $B C B C \subseteq C$.

Therefore $C B C B$ is a left quasi- interior ideal of $M$.

Since $C$ is a left quasi- interior ideal of $B$,

$$
\begin{aligned}
& C B C B=B \\
\Rightarrow & B=C B C B \subseteq C \\
\Rightarrow & B=C .
\end{aligned}
$$

Conversely suppose that $B$ is a left quasi- interior simple subsemigroup of $M$. Let $C$ be a left quasi- interior ideal of $M$ and $C \subseteq B$.

$$
\begin{aligned}
& \Rightarrow B C B C \subseteq M C M C \subseteq M B M B \subseteq B, \\
& \Rightarrow B=C, \text { since } B \text { is a quasi- interior simple semigroup. }
\end{aligned}
$$

Hence $B$ is a minimal left quasi- interior ideal of $M$.

Theorem 3.50. Let $M$ be a semigroup and $B=L L$, where $L$ is a minimal left ideal of $M$. Then $B$ is a minimal left quasi- interior ideal of $M$. 
Proof. Obviously $B=L L$ is a left quasi- interior ideal of $M$. Let $A$ be a left quasi- interior ideal of $M$ such that $A \subseteq B$.

We have $M A$ is a left ideal. Then

$$
\begin{aligned}
M A & \subseteq M B \\
& =M L L \\
& \subseteq L, \text { since } L \text { is a left ideal of } M .
\end{aligned}
$$

Therefore $M A=L$,

$$
\text { Hence } B=M A M A
$$

$$
\subseteq A
$$

Therefore $A=B$. Hence $B$ is a minimal left quasi- interior ideal of $M$.

Corollary 3.51. Let $M$ be a semigroup and $B=R R$, where $R$ is a minimal right ideal of $M$. Then $B$ is a minimal right quasi-interior ideal of $M$.

Corollary 3.52. Let $M$ be a semigroup and $B=I I$, where $I$ is a minimal ideal of $M$. Then $B$ is a minimal quasi-interior ideal of $M$.

We characterize regular semigroup using left quasi- interior ideals of semigroup.

Theorem 3.53. Let $M$ be a regular semigroup. Then every left quasi-interior ideal of $M$ is an ideal of $M$.

Proof. Let $B$ be a left quasi- interior ideal of $M$. Then

$$
\begin{aligned}
& M B M B \subseteq B \\
\Rightarrow & B \subseteq B M B, \text { since } M \text { is regular } \\
\Rightarrow & M B \subseteq M B M B \subseteq B .
\end{aligned}
$$

Similarly, we can show that $B M \subseteq M B M B \subseteq B$.

Hence the theorem.

Theorem 3.54. $M$ is a regular semigroup if and only if $A B=A \cap B$ for any right ideal $A$ and left ideal $B$ of a semigroup $M$. 
Theorem 3.55. Let $M$ be a regular semigroup. Then $B$ is a quasi-interior ideal of $M$ if and only if $M B M B=B$, and $B M B M=B$, for all quasi- interior ideals $B$ of $M$.

Proof. Suppose $M$ is a regular semigroup, $B$ is a left quasi-interior ideal of $M$ and $x \in B$. Then $M B M B \subseteq B$ and there exists $y \in M$, such that $x=x y x \in B M B$ and $B M B \subseteq M B M B$. Therefore $x \in M B M B$.

Hence $M B M B=B$. Similarly we can show that $B M B M=B$. Conversely suppose that $M B M B=B$, and $B M B M=B$, for all quasi- interior ideals $B$ of $M$.

Let $B=R \cap L$, where $R$ is a right ideal and $L$ is a left ideal of $M$.

Then $B$ is a quasi-interior ideal of $M$.

Therefore $M(R \cap L) M(R \cap L)=R \cap L$

$$
\begin{aligned}
R \cap L & =(R \cap L) M(R \cap L) M \\
& \subseteq R M L M \\
& \subseteq R L M \\
R \cap L & =(R \cap L) M(R \cap L) M \subseteq R L M R L M \subseteq R L \\
& \subseteq R \cap L \text { (since } R L \subseteq L \text { and } R L \subseteq R) .
\end{aligned}
$$

Therefore $R \cap L=R L$. Hence $M$ is a regular semigroup.

\section{WEAK-INTERIOR IDEALS OF SEMIGROUPS}

In this section, we introduce the notion of weak-interior ideal as a generalization of quasiideal and interior ideal of semigroup and study the properties of weak-interior ideal of semigroup.Throughout this paper $M$ is a semigroup with unity element.

Definition 4.1. A non-empty subset $B$ of a semigroup $M$ is said to be left weak-interior ideal of $M$ if $B$ is a subsemigroup of $M$ and $M B B \subseteq B$.

Definition 4.2. A non-empty subset $B$ of a semigroup $M$ is said to be right weak-interior ideal of $M$ if $B$ is a subsemigroup of $M$ and $B B M \subseteq B$.

Definition 4.3. A non-empty subset $B$ of a semigroup $M$ is said to be weak-interior ideal of $M$ if $B$ is a subsemigroup of $M$ and $B$ is left and right weak-interior ideal of $M$.

Example 4.4. Let $Q$ be the set of all rational numbers, $M=\left\{\left(\begin{array}{ll}0 & b \\ 0 & d\end{array}\right) \mid b, d \in Q\right\}$ be the set of matrices. The binary operation $A B$ is defined as usual matrix multiplication of $A, B$, for 
all $A, B \in M$.Then $\mathrm{M}$ is a semigroup If $R=\left\{\left(\begin{array}{ll}0 & b \\ 0 & 0\end{array}\right) \mid 0 \neq b \in Q\right\}$ then $R$ is a left weak interior ideal of the semigroup $M$ and $R$ is neither a left ideal nor a right ideal, not a weak interior ideal and not a interior ideal of the semigroup $M$.

In the following theorems, we mention some important properties and we omit the proofs since they are straight forward.

Theorem 4.5. Let $M$ be a semigroup. Then the following are hold.

(1) Every left ideal is a left weak-interior ideal of $M$.

(2) Every right ideal is a right weak-interior ideal of $M$.

(3) Every quasi ideal is a weak-interior ideal of $M$.

(4) Every ideal is a weak-interior ideal of $M$.

(5) If $B$ is a weak-interior ideal and $T$ is a subsemigroup of $M$ then $B \cap T$ is a weak-interior ideal of ring $M$.

(6) If $L$ is a left ideal and $R$ is a right ideal of a semigroup $M$ then $B=L R$ is a weak-interior ideal of $M$.

(7) $M$ is a left weak-interior simple semigroup if and only if

$<a>=M$, for all $a \in M$ and where $<a>$ is the smallest left weak-interior ideal generated by $a$.

Theorem 4.6. Let $M$ be a semigroup. Then the following are hold.

(1) If $B$ be an interior ideal of a semigroup $M$, then $B$ is a left (right) weak-interior ideal of $M$.

(2) The intersection of a left weak-interior ideal $B$ of a semigroup $M$ and a right ideal $A$ of $M$ is always a left weak-interior ideal of $M$.

(3) Let $A$ and $C$ be subsemigroup sof a semigroup $M$ and $B=A C$ and $B$ is subsemigroup of $M$. If $A$ is the left ideal of $M$ then $B$ is a weak-interior ideal of $M$.

(4) Let $M$ be a semigroup and $T$ be a non-empty subset of $M$. If subsemigroup $B$ of $M$ containing $M T T$ and $B \subseteq T$ then $B$ is a left weak-interior ideal of semigroup $M$.

(5) If $B$ is a left weak-interior ideal of semigroup $M, B T$ is an additively subsemigroup of $M$ and $T \subseteq B$ then $B T$ is a left weak-interior ideal of $M$.

(6) Let $M$ be a semigroup and $T$ be a subsemigroup of $M$. Then every subsemigroup of $T$ containing MTT is a left weak-interior ideal of $M$. 
(7) The intersection of $\left\{B_{\lambda} \mid \lambda \in A\right\}$ left weak-interior ideals of a semigroup $M$ is a left weakinterior ideal of $M$.

(8) Let $M$ be a semigroup and $B$ be a subsemigroup of $M$.If $M M B \subseteq B$ and $B M M \subseteq B$ then $B$ is a weak-interior ideal of $M$.

Theorem 4.7. Let $M$ be a semigroup and $B$ be a subsemigroup of $M . B$ is a left weak-interior ideal of $M$ if and only if there exists left ideal $L$ such that $L L \subseteq B \subseteq L$.

Proof. Suppose $B$ is a left weak-interior ideal of the semigroup $M$.Then $M B B \subseteq B$. Let $L=M B$. Then $L$ is a left ideal of $M$. Therefore $L L \subseteq B \subseteq L$.Conversely suppose that there exists left ideal $L$ of $M$ such that $L L \subseteq B \subseteq L$.Then $M B B \subseteq M L L \subseteq B$.Hence $B$ is a left weak-interior ideal of $M$.

Corollary 4.8. Let $M$ be a semigroup and $B$ be a subsemigroup of $M$. B is a weak-interior ideal of $M$ if and only if there exist ideal $R$ such that $R R \subseteq B \subseteq R$. .

Theorem 4.9. Let $A$ and $C$ be left weak-interior ideals of a semigroup $M$, and $B$ is subsemigroup of M. If $B=A C=C A$ then $B$ is a left quasi-interior ideal of $M$.

Proof. Let $A$ and $C$ be left weak-interior ideals of the semigroup $M$ and $B=A C$.

Then $B B=A C A C=A C C C A C \subseteq A C M C M C \subseteq A C=B$. Therefore $B=A C$ is a subsemigroup of $M$.Then $M B B=M A C A C \subseteq M A A C \subseteq A C=B$. Hence $B$ is a left weak-interior ideal of $M$.

Theorem 4.10. Let $M$ be a semigroup. If $M=M a$, for all $a \in M$. Then every left weak-interior ideal of $M$ is a quasi ideal of $M$.

Proof. Let $B$ be a left weak-interior ideal of the semigroup $M$ and $a \in B$. Then $\Rightarrow M a \subseteq$ $M B, \Rightarrow M \subseteq M B \subseteq M, \Rightarrow M B=M, \Rightarrow M B=M B B \subseteq B, \Rightarrow M B \cap B M \subseteq B \cap B M \subseteq B$. Therefore $B$ is a quasi ideal of $M$. Hence the theorem.

Theorem 4.11. $B$ is a left weak-interior ideal of a semigroup $M$ if and only if $B$ is a left ideal of some left ideal of a semigroup $M$.

Proof. Suppose $B$ is a left ideal of left ideal $R$ of the semigroup $M$. Then $R B \subseteq B, M R \subseteq R$ and $M B B \subseteq M R B \subseteq R B \subseteq B$. Therefore $B$ is a left weak-interior ideal of a semigroup $M$. Conversely suppose that $B$ is a left weak-interior ideal of a semigroup $M$. Then $M B B \subseteq B$. Therefore $B$ is a left ideal of left ideal $M B$ of the semigroup $M$. 
Definition 4.12. A semigroup $M$ is a left (right) simple semigroup if $M$ has no proper left (right) ideals of $M$.

Definition 4.13. A semigroup $M$ is said to be simple semigroup if $M$ has no proper ideals of $M$.

Definition 4.14. A semigroup $M$ is said to be left(right) weak-interior simple semigroup if $M$ has no left(right) weak-interior ideal other than $M$ itself.

Definition 4.15. A semigroup $M$ is said to be weak-interior simple semigroup if $M$ has no weak-interior ideal other than $M$ itself.

Theorem 4.16. If $M$ is a semigroup and every non zero element has inverse, then $M$ is a left weakinterior simple semigroup.

Proof. Let $B$ be a proper left weak-interior ideal of the semigroup $M, x \in M$ and $0 \neq a \in B$. There exists $b \in M$ such that $a b=1$. Therefore $a b x=x . x \in B M$ and $M \subseteq B M$. We have $B M \subseteq M$. Hence $M=B M$. Similarly we can prove $M B=M . M=M B=M B B \subseteq B, M \subseteq B$. Therefore $M=B$. Hence the theorem.

Theorem 4.17. Let $M$ be a left simple semigroup. Every left weak-interior ideal of $M$ is an ideal of $M$. Proof. Let $M$ be a left simple semigroup and $B$ be a left weak-interior ideal of $M$. Then $M B B \subseteq$ $B$ and $M B$ is a left ideal of $M$. Since $M$ is a left simple semigroup, we have $M B=M$. Therefore $M B \subseteq M B B \subseteq B$. Then $B M=B M B=B M B B \subseteq B B \subseteq B$. Hence every left weak-interior ideal is an ideal of $M$.

Theorem 4.18. Let $M$ be a semigroup. Then $M$ is a left weak-interior simple semigroup if and only if $M a a=M$, for all $a \in M$.

Proof. Suppose $M$ is the left-quasi interior simple semigroup and $a \in M$. Then $M a a$ is a weak-interior ideal of $M$. Hence $M a a=M$, for all $a \in M$.

Conversely suppose that $M a a=M$, for all $a \in M$. Let $B$ be a left weak-interior ideal of the semigroup $M$ and $a \in B . M=M a a \subseteq M B B \subseteq B$. Therefore $M=B$. Hence $M$ is a left weak-interior simple semigroup.

Theorem 4.19. Let $M$ be a semigroup and $B$ be a left weak-interior ideal of $M$. Then $B$ is a minimal left weak-interior ideal of $M$ if and only if $B$ is a left weak-interior simple subsemigroup of $M$. 
Proof. Let $B$ be a minimal left weak-interior ideal of the semigroup $M$ and $C$ be a left weakinterior ideal of $B$. Then $B C C \subseteq C$.

and $B C C$ is a left weak-interior ideal of $M$.

Since $B$ is a minimal weak-interior ideal of $M, B C C=B, \Rightarrow B=B C C \subseteq C, \Rightarrow B=C$. Conversely suppose that $B$ is the left weak-interior simple subsemigroup of $M$. Let $C$ be a left weak-interior ideal of $M$ and $C \subseteq B . \Rightarrow B C C \subseteq M C C \subseteq M B B \subseteq B$.Therefore $C$ is a left weak-interior of $B . \Rightarrow B=C$,since $B$ is a left weak-interior simple subsemigroup of $M$. Hence $B$ is a minimal left weak-interior ideal of $M$.

Theorem 4.20. Let $M$ be a semigroup, any left weak-interior ideal of $M$ commutes with $M$ and $B=L L$, where $L$ is a minimal left ideal of $M$. Then $B$ is a minimal left weak-interior ideal of $M$.

Proof. Obviously $B=L L$ is a left weak-interior ideal of $M$. Let $A$ be a left weak-interior ideal of $M$ such that $A \subseteq B$.

We have $M A$ is a left ideal of $M$. Then $M A \subseteq M B=M L L \subseteq L$, since $L$ is a left ideal of $M$. Therefore $M A=L$, since $L$ is a minimal left ideal of $M$. Hence $B=M A M A \subseteq M M A A \subseteq$ $M A A \subseteq A$. Therefore $A=B$. Hence $B$ is a minimal left weak-interior ideal of $M$.

Theorem 4.21. Let $M$ be semigroup Then $B$ is a weak-interior ideal of an idempotent regular semigroup $M$ if and only if $B B M=B$ and $M B B=B$ for all weak-interior ideals $B$ of $M$.

Proof. Suppose $M$ is the regular semigroup, $B$ is the weak-interior ideal of $M$ and $x \in B$.Then $M B B \subseteq B, y \in M$, such that $x=x x=x x y x x \in M B B$. Therefore $x \in M B B$. Hence $M B B=B$. Similarly we can prove $B B M=B$. Conversely suppose tha xt $B B M=B$ and $M B B=B$ for all weak-interior ideals $B$ of $M$. Let $B=R \cap L$ where $R$ is a right ideal and $L$ is a left ideal of $M$. Then $B$ is a weak-interior ideal of $M$.

Therefore $(R \cap L)(R \cap L) M=R \cap L, R \cap L=(R \cap L)(R \cap L) M \subseteq R R M, R \cap L=M(R \cap$ $L)(R \cap L) \subseteq M L L, R \cap L=(R \cap L)(R \cap L) M \subseteq R R M M L L \subseteq R L$, since $R L \subseteq L$ and $R L \subseteq R, R L \subseteq R \cap L$. Therefore $R \cap L=R L$. Hence $M$ is a regular semigroup.

\section{Conclusion}

As a further generalization of ideals, we introduced the notion of quasi-interior ideals and weak -interior ideals as a generalization of quasi ideal, bi-ideal and interior ideal of semigroups.We study the properties of quasi-interior ideals and weak-interior ideals of semigroups and characterized the quasi-interior ( weak-interior ) simple semigroup, regular semigroup 
using quasi-interior ideals and weak-interior ideal ideals of semigroups. In continuity of this paper, we study prime, maximal and minimal quasi- interior ( weak-interior ) ideals of semigroups.

\section{REFERENCES}

[1] R. A. Good and D. R. Hughes, Associated groups for a semigroup, Bull. Amer. Math. Soc., 58 (1952), 624-625.

[2] M. Henriksen, Ideals in semirings with commutative addition, Amer. Math. Soc. Notices, 5 (1958), 321.

[3] K. Iseki, Quasi-ideals in semirings without zero, Proc. Japan Acad., 34 (1958), 79-84.

[4] K. Iseki, Ideal theory of semiring, Proc. Japan Acad., 32 (1956), 554-559.

[5] K. Iseki, Ideal in semirings Proc. Japan Acad., 34 (1958), 29-31.

[6] K. Izuka, On the Jacobson radical of a semiring, Tohoku, Math. J., 11(2) (1959), 409-421.

[7] R. D. Jagatap, Y.S. Pawar, Quasi-ideals and minimal quasi-ideals in semirings, Novi Sad J. Math., 39(2) (2009), 79-87.

[8] S. Lajos, (m;k;n)-ideals in semigroups. In: Notes on Semigroups II, Karl Marx Univ. Econ., Dept. Math. Budapest, 1 (1976), 12-19.

[9] S. Lajos On the bi-ideals in semigroups, Proc. Japan Acad., 45 (1969), 710-712.

[10] S. Lajos and F. A. Szasz, On the bi-ideals in associative ring, Proc. Japan Acad., 46 (1970), 505-507.

[11] Marapureddy Murali Krishna Rao, Bi -interior ideals of semigroups,Discussiones Math. Gen. Alg. Appl. 38 (2018), 69-70.

[12] M. Murali Krishna Rao, Left bi-quasi ideals of semirings, Bull. Int. Math. Virtual Inst., 8 (2018), 45-53.

[13] Marapureddy Murali Krishna Rao. Bi-quasi-ideals and fuzzy bi-quasi ideals of semigroups. Bull. Int. Math. Virtual Inst., 7 (2) (2017), 231-242.

[14] M. Murali Krishna Rao, $\Gamma$-semirings-I, Southeast Asian Bull. Math., 19 (1) (1995), 49-54.

[15] M. Murali Krishna Rao, Г-semirings-II, Southeast Asian Bulletin of Mathematics, 21 (3) (1997), 281-287.

[16] M. Murali Krishna Rao, The Jacobson radical of $\Gamma$-semiring, Southeast Asian Bull. Math., 23 (1999), 127-134.

[17] M. Murali Krishna Rao and B. Venkateswarlu, Regular $\Gamma$-incline and field $\Gamma$-semiring, Novi Sad J. Math., 45 (2) (2015), 155-171.

[18] M. Murali Krishna Rao, $\Gamma-$ semiring with identity, Disc. Math. Gen. Alg. Appl., 37 (2017), 189-207. doi:10.7151/dmgaa.1276.

[19] M. Murali Krishna Rao, Ideals in ordered $\Gamma$-semirings, Disc. Math. Gen. Alg. Appl., 38(2018), 47-68.

[20] M. Murali Krishna Rao, Bi-interior Ideals in semigroups, Disc. Math. Gen. Alg. and Appl., 38 (2018), 69-78.

[21] M. Murali Krishna Rao, Left bi-quasi ideals of semirings, Bull. Int. Math. Virtual Inst., 8 (2018), 45-53.

[22] M.Murali Krishna Rao, Bi-quasi-ideals and fuzzy bi-quasi ideals of $\Gamma$-semigroups, Bull. Int. Math. Virtual Inst., 7 (2) (2017), 231-242. 
[23] Marapureddy Murali Krishna Rao, A study of bi-quasi interior ideal as a new generalization of ideal of generalization of semiring, Bull. Int. Math. Virtual Inst, 8 (2018), 519-535.

[24] M.Murali Krishna Rao, Quasi- interior ideals of semirings, Bull. Int. Math. Virtual Inst., 9 (1) (2018), $231-242$.

[25] M. Murali Krishna Rao, A study of generalization of bi- ideal, quasi- ideal and interior ideal of semigroup, Math. Morovica, 22 (2) (2018), 103-115.

[26] M. Murali Krishna Rao, B. Venkateswarlu and N.Rafi, Left bi-quasi-ideals of $\Gamma$-semirings, Asia Pac. J. Math., 4( 2) (2017), 144-153.

[27] M. Murali Krishna Rao and B. Venkateswarlu, Bi-interior Ideals in $\Gamma$ - semirings , Disc. Math. Gen. Alg. and Appl., 38(2) (2018), 209-221.

[28] M. Murali Krishna Rao and B. Venkateswarlu, Right derivation of ordered $\Gamma$-semirings, Disc. Math. Gen. Alg. Appl., 36 (2016), 209-221.

[29] A. M. Shabir , A Batod, A note on quasi ideal in semigroups, Southeast Asian Bull. Math., 7 (2004), 923-928.

[30] O. Steinfeld, Uher die quasi ideals, Von halbgruppend Publ. Math., Debrecen, 4 (1956), 262-275. 